\title{
Diagnostic performance of point-of-use ultrasound of resuscitation outcomes: A systematic review and meta-analysis of 3265 patients
}

\author{
Maciej Dudek ${ }^{1}$, Lukasz Szarpak ${ }^{1,2}$ (®) Frank W. Peacock ${ }^{3}$, Aleksandra Gasecka ${ }^{4,5}$, \\ Tomasz Michalski ${ }^{6}$, Pawel Wroblewski ${ }^{7}$, Halla Kaminska ${ }^{8}$, Gabriela Borkowska ${ }^{9}$, \\ Ewa Skrzypek ${ }^{10}$, Adam Smereka ${ }^{11}$, Jaroslaw Meyer-Szary ${ }^{12}$, \\ Sylwia Marciniak ${ }^{13}$, Mariola Malecka ${ }^{1,14}$
}

${ }^{1}$ Polish Society of Disaster Medicine, Warsaw, Poland; ${ }^{2}$ Maria Sklodowska-Curie Bialystok Oncology Center, Bialystok, Poland; ${ }^{3}$ Henry JN Taub Department of Emergency Medicine, Baylor College of Medicine Houston, Texas, United States; ${ }^{4} 1^{\text {st }}$ Chair and Department of Cardiology, Medical University of Warsaw,

Poland; ${ }^{5}$ Department of Cardiology, University Medical Center Utrecht, The Netherlands;

${ }^{6} 1^{\text {st }}$ Department of Cardiology, Medical University of Gdansk, Poland; ${ }^{7}$ Department of Emergency Medical

Service, Wroclaw Medical University, Wroclaw, Poland; ${ }^{8}$ Department of Pediatrics and Children's Diabetology, Faculty of Medical Sciences in Katowice, Medical University of Silesia, Zabrze, Poland;

${ }^{9}$ Faculty of Medicine, Collegium Medicum, Cardinal Stefan Wyszynski University, Warsaw, Poland; ${ }^{10}$ Department of History of Medicine, Medical University of Warsaw, Poland; ${ }^{11}$ Department of Gastroenterology and Hepatology, Faculty of Medicine, Wroclaw Medical University, Wroclaw, Poland; ${ }^{12}$ Department of Pediatric Cardiology and Congenital Heart Diseases, Medical University of Gdansk, Poland; ${ }^{13}$ Students Research Club, Maria Sklodowska-Curie Medical Academy in Warsaw, Poland; ${ }^{14}$ Institute of Outcomes Research, Maria Sklodowska-Curie Medical Academy in Warsaw, Poland

\begin{abstract}
Background: Echocardiography in the setting of resuscitation can provide information as to the cause of the cardiac arrest, as well as indicators of futility. This systematic review and meta-analysis were performed to determine the value of point-of-care ultrasonography (PoCUS) in the assessment of survival for adult patients with cardiac arrest.

Methods: This meta-analysis was performed in adherence to the Preferred Reporting Items for Systematic Reviews and Meta-Analyses (PRISMA) guidelines. PubMed, EMBASE, Web of Science, Cochrane have been searched from databases inception until March $2^{\text {nd }} 2021$. The search was limited to adult patients with cardiac arrest and without publication dates or country restrictions. Papers were chosen if they met the required criteria relating to the sensitivity, specificity, accuracy, positive predictive value, and negative predictive value of this diagnostic technique concerning resuscitation outcomes.

Results: This systematic review identified 20 studies. Overall, for survival to hospital discharge, PoCUS was 6.2\% sensitivity (95\% confidence interval [CI] 4.7-8.0\%) and $2.1 \%$ specific (95\% CI 0.8-4.2\%). PoCUS sensitivity and specificity for return of spontaneous circulation were $23.8 \%$ (95\% CI 21.4-26.4\%) and 50.7\% (95\% CI 45.8-55.7\%) respectively, and for survival to admission $13.8 \%$ (95\% CI 12.2-15.5\%) and 20.1\% (95\% CI 16.2-24.3\%), respectively.

Conclusions: The results do not allow unambiguous recommendation of PoCUS as a predictor of resuscitation outcomes and further studies based on a large number of patients with full standardization of operators, their training and procedures performed were necessary. (Cardiol J 2023; 30, 2: 237-246)

Key words: cardiac arrest, ultrasonography, echocardiography, cardiopulmonary resuscitation, outcome, systematic review, meta-analysis

Address for correspondence: Ewa Skrzypek, MD, PhD, Department of History of Medicine, Medical University of Warsaw, ul. Żwirki i Wigury 61, 02-091 Warszawa, Poland, tel: +48 604075561, e-mail: ewa.skrzypek@wum.edu.pl

Received: 7.03.2021 Accepted: 11.03.2021 Early publication date: 23.04.2021

This article is available in open access under Creative Common Attribution-Non-Commercial-No Derivatives 4.0 International (CC BY-NC-ND 4.0) license, allowing to download articles and share them with others as long as they credit the authors and the publisher, but without permission to change them in any way or use them commercially.
\end{abstract}




\section{Introduction}

Echocardiography in the setting of cardiopulmonary resuscitation (CPR) can provide information as to the cause of the sudden cardiac arrest (SCA), as well as indicators of futility [1-3]. In the first application, echocardiography can identify potentially reversible causes of arrest. This procedure is performed by use of the subcostal, apical and parasternal views to identify cardiac tamponade, findings suggestive of pulmonary embolism, and a pleural view to identify pneumothorax [4-6]. The diagnosis of pulmonary embolism may be challenging, as findings of isolated right ventricular dilation must be considered with caution. Right ventricular dilation occurs within minutes of cardiac arrest, as blood shifts from the systemic circulation to the right heart along its pressure gradient $[7,8]$, which has been uniformly reported in porcine models of cardiac arrest, regardless of causes that included hypovolemia, hyperkalemia, and primary arrhythmia $[9,10]$.

The second most useful application of echocardiography during $\mathrm{CPR}$ is that of determining the probability of a successful resuscitation. In this situation echocardiography is used to identify spontaneous cardiac movement $[11,12]$. The metaanalysis conducted by Tsou et al. [11] has revealed that spontaneous cardiac movement possesses a sensitivity and specificity of $95 \%$ and $80 \%$ (95\% confidence interval [CI] 0.72-0.99, and 0.63-0.91, respectively) for predicting return of spontaneous circulation (ROSC) during cardiac arrest, with a positive and negative likelihood ratio of 4.8 and 0.06 (95\% CI 2.5-9.4, and 0.01-0.39, respectively). Despite these findings, the 2020 International Consensus on Cardiopulmonary Resuscitation and Emergency Cardiovascular Care Science with Treatment Recommendations [13] suggested against the use of point-of-care ultrasound (PoCUS) for prognostication during CPR. This occurred because the overall certainty of evidence was rated as very low for all outcomes, primarily due to the risk of bias, inconsistency, and/or imprecision.

Finally, despite the suggested somewhat controversial value of echocardiography in the course of resuscitation, clinicians may have reservations to adopt because of interference with cardiac compressions leading to ineffectual CPR. To address this, some [14] have suggested protocols to perform echocardiography simultaneously with the rhythm check, thus minimizing interruptions to chest compressions. Whether or not a practitioner decides to use echocardiography during a resuscita- tion, ultimately its use is subject to availability of equipment and skilled operators. Although rapidly becoming more available, the pre-hospital application of PoCUS is still commonly limited.

Because of these concerns and challenges, we sought to evaluate the current status of PoCUS as an aid to diagnosis and a determinate of prognosis during resuscitation. The purpose was to review and appraise the diagnostic accuracy of echocardiography in CPR. A priori subgroup analyses of the primary outcome were planned for location: out-of-hospital cardiac arrest (OHCA) versus in-hospital cardiac arrest (IHCA).

\section{Methods}

This systematic review and meta-analysis were performed in accordance with the Preferred Reporting Items for Systematic reviews and Meta-Analysis (PRISMA) statement [15].

\section{Data sources and searches}

Two authors independently (M.D. and H.K.) conducted a search of PubMed, Scopus, EMBASE, Web of Science, and the Cochrane Central Register and Controlled Trials (CENTRAL) database from inception to March $2^{\text {nd }}, 2021$, with the following search strategy: "out-of-hospital cardiac arrest" OR "OHCA" OR "in-hospital cardiac arrest" OR "IHCA" OR "cardiac arrest" OR "heart arrest" OR "heart attack" OR "advanced life support" OR "resuscitation" OR "CPR" AND "cardiac ultrasound" OR "cardiac ultrasonography" OR "heart ultrasound" OR "heart ultrasonography" OR "echocardiography" OR "ultrasound imaging” OR "USG” OR "US” OR “ultrasound” OR "ultrasonographic" OR "echocardiogram" OR "point-of-care ultrasonography” OR "PoCUS" OR point-of-care echocardiography". Only studies published in English were included in the meta-analysis. However, the search was limited to human studies without publication date, or country restrictions. Gray literature repositories such as Google Scholar were also searched. Finally, further reviewed references to echocardiography in eligible articles and systematic reviews were manually retrieved.

\section{Selection criteria}

Two investigators (M.D. and H.K.) evaluated independently all relevant studies for eligibility criteria and pooled analysis. Disagreements between the authors regarding values or analysis assignments were resolved through discussion with a third researcher (L.S.), and the decision 
was taken by the majority of the researchers. Raw data were extracted by using a standardized, premade form. Care was taken to avoid inclusion of data from duplicate publications. In any case of suspected data discrepancies, the relevant author was contacted directly.

Studies included in this meta-analysis fulfilled the following criteria (PICOS): (P) Population: adults with cardiac arrest; (I) Intervention: point-of-care echocardiography during CPR; (C) Comparator: absence of that finding or a different finding on point-of-care echocardiography during resuscitation procedure; (O) Outcome: prognosticate clinical outcome: ROSC, survival to hospital admission or survival to hospital discharge; (S) Study design: randomized controlled trials (RCTs) or non-RCTs.

Studies that enrolled children and animal studies were excluded. Case reports, case series, guidelines, review articles; consensus statements, editorials, letters, conference abstracts, studies not pertaining to the field of inters or studies with insufficient data for reconstruction $2 \times 2$ table were also excluded.

\section{Data extraction}

Data were independently extracted by two reviewers (M.D. and L.S.) and were verified by a third reviewer (L.S.). The following data categories were extracted from the included studies: study population characteristics, operator type, ultrasonography window type; initial rhythm, resuscitation outcomes including ROSC, survival to hospital admission or survival to hospital discharge. True positive, false positive, false negative, and true negative numbers of echocardiography were obtained. The results were summarized in 2-by-2 contingency tables.

\section{Quality assessment}

Two investigators (A.G. and H.K.) independently extracted individual study data and evaluated studies for risk of bias. Any disagreements were discussed and resolved in a consensus meeting with the third reviewer (M.M.). The ROBINS-I tool (tool to assess risk of bias in non-randomized studies of interventions) was used to assess the quality of non-randomized trials [16] and the RoB 2 tool (revised tool for risk of bias in randomized trials) was used to assess the quality of randomized studies [17]. The Robvis application was used to visualize risk of bias assessments [18]. The scale has seven main domains (confounding, participant selection, classification of interventions, deviation from interventions, missing data, outcome measurement, and selection of reported results) and assigns one point for each of the following four judgements: critical, serious, moderate, and low. The review authors' judgments about each risk of bias items are provided in Supplementary Figures S1 and S2.

\section{Outcomes}

The primary outcome of the current metaanalysis was survival to hospital discharge or 30-day survival. The secondary outcomes were return of spontaneous circulation or survival to hospital admission in case of OHCA.

\section{Data analysis}

All statistical analyses were performed using STATA and Review Manager Software 5.4 (The Cochrane Collaboration, Oxford, Copenhagen, Denmark). Sensitivity, specificity, positive likelihood ratio $(+\mathrm{LR})$ and negative $\mathrm{LR}(-\mathrm{LR})$, and the log diagnostic odds ratio, including the $95 \% \mathrm{CI}$, were calculated. The primary objective was to estimate pooled measurements of diagnostic accuracy: pooled sensitivity and specificity using the Mantel-Haenszel odds ratios [19], and pooled positive and negative LR using the DerSimonian-Laird method [20]. An overall area under the receiver-operating-characteristic (ROC) curve was also calculated. Heterogeneity was assessed using the Cochran Q-statistic ( $\mathrm{p}$ less than 0.05 indicated the presence of heterogeneity) and the inconsistency $\left(\mathrm{I}^{2}\right)$ test [21]. According to Higgins a p-value of $<0.10$ or $\mathrm{I}^{2}$ statistic of $>50 \%$ indicated substantial statistical heterogeneity [21]. Subgroup sensitivity analyses were also conducted to determine the robustness of findings. Forest plots were prepared and performed with Review Manager Software 5.4 (The Cochrane Collaboration, Oxford, Copenhagen, Denmark).

\section{Results}

\section{Search results}

Figure 1 shows a flow diagram summarizing the literature search. A total of 6217 studies were identified during the initial search. After removing 1115 duplicates, 5102 titles and abstracts were reviewed and then 5060 studies were excluded. After reviewing the full text of 47 eligible articles, finally 20 original research articles [22-41] including a total of 3265 patients were included in this meta-analysis. Study types included prospective cohort design, retrospective and case-control studies. The publication dates of these studies ranged from 2001 


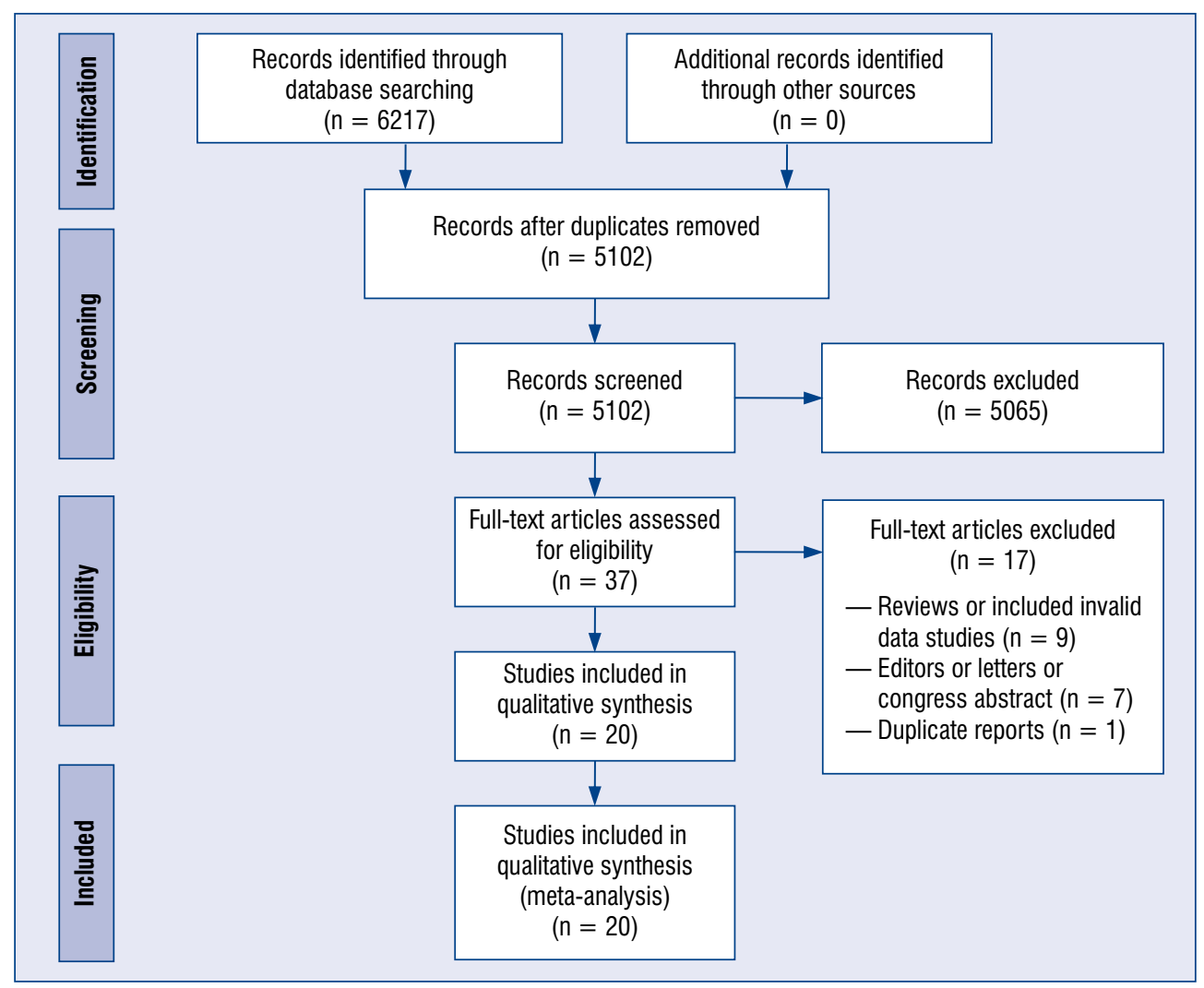

Figure 1. Meta-analysis flow chart of included and excluded studies.

to 2019. Six studies were conducted in the United States [25, 30, 37-40], 3 in Turkey [27, 36, 41], 2 in Canada [22, 24], and 1 in each of the following countries: Austria [23], Germany [26], Iran [28], Singapore [29], Brazil [31], United Kingdom [33], Republic of Korea [34], and Taiwan [35]. One study was also conducted as a multi-country trial.

\section{Characteristics and quality assessment of included studies}

Table 1 lists the study and population characteristics. The number of patients in the study ranged from 20 to 793 . Mean age ranged from 48.6 to 71.1 years. The study's ultrasonographic characteristics, as well as methodology characteristics, are summarized in Supplementary Table S1 and S2. Results of the quality assessment of studies are summarized in Supplementary Figures S1 and S2.

\section{Primary outcome}

Survival to hospital discharge was reported in 6 studies [22-24, 31, 32, 36], in which sensitivity values ranged from 0.01 to 0.46 and specificity values ranged from 0.00 to 0.05 (Fig. 2). The summary sensitivity and specificity values were 0.062
$(0.047,0.078)$ and $0.021(0.008,0.042)$, respectively. The $\mathrm{Q}$ test revealed significant heterogeneity $(\mathrm{Q}=24.54 ; \mathrm{p}<0.001)$, with substantial heterogeneity detected for sensitivity $\left(\mathrm{I}^{2}=97.5 \%\right.$; $\mathrm{p}<0.001)$ and specificity $\left(\mathrm{I}^{2}=30.9 \% ; \mathrm{p}=0.215\right)$. The area under the hierarchic summary ROC curve was 0.0112 .

Subgroup analysis showed that sensitivity and specificity of echocardiographic test related to survival to hospital discharge in the OHCA group was: 0.041 (95\% CI 0.018-0.080) and 0.024 (95\% CI 0.001-0.129), respectively (Suppl. Table S3).

\section{Secondary outcomes}

The 9 studies reported return of spontaneous circulation [22, $24,28,31,32,34,36,41]$, in which pooled results of sensitivity and specificity were 0.238 (95\% CI 0.214-0.264) and 0.507 (95\% CI 0.458-0.557), respectively (Fig. 3). The $\mathrm{Q}$ test revealed significant heterogeneity $(\mathrm{Q}=35.779$; $\mathrm{p}<0.001$ ), with substantial heterogeneity detected for sensitivity $\left(\mathrm{I}^{2}=85.5 \%\right.$; $\left.\mathrm{p}<0.001\right)$ and specificity $\left(\mathrm{I}^{2}=93.8 \% ; \mathrm{p}<0.001\right)$. The area under the hierarchic summary ROC curve was 0.3296 .

Use of PoCUS in cases of OHCA arrest was associated with a study sensitivity of 0.194 (95\% CI 
Table 1. Characteristics of the included studies.

\begin{tabular}{|c|c|c|c|c|c|c|}
\hline Study & Country & $\begin{array}{l}\text { Study } \\
\text { desing }\end{array}$ & $\begin{array}{c}\text { Cardiac } \\
\text { arrest setting }\end{array}$ & $\begin{array}{l}\text { No. of } \\
\text { patients }\end{array}$ & $\begin{array}{l}\text { Age (mean } \\
\pm \text { SD) }\end{array}$ & $\begin{array}{l}\text { Inithial } \\
\text { rhythm }\end{array}$ \\
\hline Aichinger et al. 2012 & Austria & $\begin{array}{c}\text { Prospective } \\
\text { observational study }\end{array}$ & OHCA & 42 & $70.3 \pm 2.4$ & $\begin{array}{c}\text { VF/pVT: } 11 \\
\text { AS: } 20 \\
\text { PEA: } 11\end{array}$ \\
\hline Atkinson et al. 2019 & Canada & Retrospective study & IHCA & 223 & $65.3 \pm 4.2$ & NS \\
\hline Backett et al. 2019 & Canada & Retrospective study & OHCA & 180 & $65.27 \pm 15.02$ & $\begin{array}{l}\text { AS: } 135 \\
\text { PEA: } 45\end{array}$ \\
\hline Blaivas et al. 2001 & USA & $\begin{array}{l}\text { Prospective observa- } \\
\text { tional study }\end{array}$ & OHCA & 173 & $71.1 \pm 2.7$ & $\begin{array}{c}\text { VF/pVT: } 66 \\
\text { AS: } 65 \\
\text { PEA: } 38\end{array}$ \\
\hline Breitkreutz et al. 2010 & Germany & $\begin{array}{l}\text { Prospective observa- } \\
\text { tional study }\end{array}$ & OHCA & 100 & $65 \pm 19$ & $\begin{array}{c}\text { VF/pVT: } 24 \\
\text { AS:38 } \\
\text { PEA: } 22\end{array}$ \\
\hline Cebicci et al. 2014 & Turkey & Retrospective study & $\begin{array}{l}\text { IHCA and } \\
\text { OHCA }\end{array}$ & 410 & $63.2 \pm 20.7$ & $\begin{array}{c}\text { VF/pVT: } 45 \\
\text { AS: } 290 \\
\text { PEA: } 75\end{array}$ \\
\hline Chardoli et al. 2012 & Iran & $\begin{array}{c}\text { Prospective } \\
\text { interventional study }\end{array}$ & IHCA & 100 & $58 \pm 6.1$ & PEA: 100 \\
\hline Chua et al. 2017 & Singapore & Prospective study & OHCA & 104 & $69.3 \pm 7.2$ & $\begin{array}{c}\text { VF/pVT: } 17 \\
\text { AS: } 47 \\
\text { PEA: } 33\end{array}$ \\
\hline Cureton et al. 2012 & USA & Retrospective study & OHCA & 318 & NS & NS \\
\hline Flato et al. 2015 & Brazil & $\begin{array}{l}\text { Prospective, } \\
\text { observational } \\
\text { cohort study }\end{array}$ & IHCA & 49 & $60.0 \pm 17.6$ & $\begin{array}{l}\text { AS: } 17 \\
\text { PEA: } 32\end{array}$ \\
\hline Gaspari et al. 2016 & $\begin{array}{l}\text { USA/ } \\
\text { /Canada }\end{array}$ & $\begin{array}{c}\text { Non-randomized, } \\
\text { prospective, protocol- } \\
\text {-driven observational } \\
\text { study }\end{array}$ & $\begin{array}{l}\text { IHCA and } \\
\text { OHCA }\end{array}$ & 793 & $64.2 \pm 17.4$ & $\begin{array}{l}\text { AS: } 379 \\
\text { PEA: } 414\end{array}$ \\
\hline Hayhurst et al. 2011 & UK & Retrospective study & $\begin{array}{l}\text { IHCA and } \\
\text { OHCA }\end{array}$ & 50 & NS & $\begin{array}{l}\text { VF/pVT: } 6 \\
\text { AS: } 20 \\
\text { PEA: } 23\end{array}$ \\
\hline Kim et al. 2016 & $\begin{array}{l}\text { Republic } \\
\text { of Korea }\end{array}$ & $\begin{array}{l}\text { Prospective clinical } \\
\text { observational study }\end{array}$ & OHCA & 48 & $63.9 \pm 14.5$ & NS \\
\hline Lien et al. 2018 & Taiwan & $\begin{array}{c}\text { Prospective } \\
\text { observational study }\end{array}$ & OHCA & 177 & $70.9 \pm 14.8$ & $\begin{array}{c}\text { VF/pVT: } 31 \\
\text { AS: } 82 \\
\text { PEA: } 64\end{array}$ \\
\hline Ozen et al. 2016 & Turkey & $\begin{array}{l}\text { Prospective, single } \\
\text { center study }\end{array}$ & $\begin{array}{l}\text { IHCA and } \\
\text { OHCA }\end{array}$ & 129 & $68.96 \pm 16.44$ & $\begin{array}{l}\text { VF/pVT: } 30 \\
\text { PEA/AS: } 20\end{array}$ \\
\hline Salen et al. 2001 & USA & $\begin{array}{l}\text { Prospective clinical } \\
\text { observation study }\end{array}$ & $\begin{array}{l}\text { IHCA and } \\
\text { OHCA }\end{array}$ & 102 & NS & $\begin{array}{c}\text { VF/pVT: } 11 \\
\text { AS: } 36 \\
\text { PEA: } 55\end{array}$ \\
\hline Salen et al. 2005 & USA & $\begin{array}{l}\text { Prospective clinical } \\
\text { observation study }\end{array}$ & $\begin{array}{l}\text { IHCA and } \\
\text { OHCA }\end{array}$ & 70 & NS & $\begin{array}{l}\text { AS: } 36 \\
\text { PEA: } 34\end{array}$ \\
\hline Schuster et al. 2009 & USA & Retrospective study & $\begin{array}{l}\text { IHCA and } \\
\text { OHCA }\end{array}$ & 28 & $48.6 \pm 20.2$ & PEA: 28 \\
\hline Tayal et al. 2003 & USA & $\begin{array}{l}\text { Observational, } \\
\text { prospective series }\end{array}$ & OHCA & 20 & $57 \pm 15$ & NS \\
\hline Tomruk et al. 2012 & Turkey & $\begin{array}{c}\text { Prospective } \\
\text { follow-up study }\end{array}$ & $\begin{array}{l}\text { IHCA and } \\
\text { OHCA }\end{array}$ & 149 & $61.6 \pm 17.9$ & $\begin{array}{l}\text { VF/pVT: } 8 \\
\text { AS: } 77 \\
\text { PEA: } 64\end{array}$ \\
\hline
\end{tabular}

AS - asystole; IHCA — in-hospital cardiac arrest; NS - not specified; OHCA — out-of-hospital cardiac arrest; PEA — pulseless electrical activity; pVT — pulseless ventricular tachycardia; SD — standard deviation; VF — ventricular fibrillation 


\begin{tabular}{|c|c|c|c|c|c|c|c|c|}
\hline Study & TP & FP & FN & TN & Sensitivity $(95 \% \mathrm{Cl})$ & Specificity $(95 \% \mathrm{Cl})$ & Sensitivity $(95 \% \mathrm{Cl})$ & Specificity $(95 \% \mathrm{Cl})$ \\
\hline Aichinger 2012 & 4 & 3 & 34 & 1 & $0.11[0.03,0.25]$ & $0.25[0.01,0.81]$ & $\rightarrow$ & $\longrightarrow$ \\
\hline Atkinson 2019 & 2 & 21 & 159 & 1 & $0.01[0.00,0.04]$ & $0.05[0.00,0.23]$ & - & $=$ \\
\hline Backett 2019 & 2 & 19 & 158 & 1 & $0.01[0.00,0.04]$ & $0.05[0.00,0.25]$ & - & $=$ \\
\hline Flato 2015 & 6 & 21 & 27 & 0 & $0.18[0.07,0.35]$ & $0.00[0.00,0.16]$ & - & E- \\
\hline Gaspari 2016 & 10 & 253 & 527 & 3 & $0.02[0.01,0.03]$ & $0.01[0.00,0.03]$ & घ & a \\
\hline Ozen 2016 & 43 & 34 & 51 & 1 & $0.46[0.35,0.56]$ & $0.03[0.00,0.15]$ & 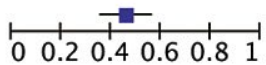 & $\begin{array}{llllll} & & 1 & 1 & 1 \\
& 0.2 & 0.4 & 0.6 & 0.8 & 1\end{array}$ \\
\hline
\end{tabular}

Figure 2. Forrest plot of the overall sensitivity and specificity of echocardiography for predicting survival to hospital discharge after cardiac arrest; $\mathrm{Cl}$ — confidence interval; TP — true positive; FP — false positive; FN — false negative; $\mathrm{TN}$ - true negative.

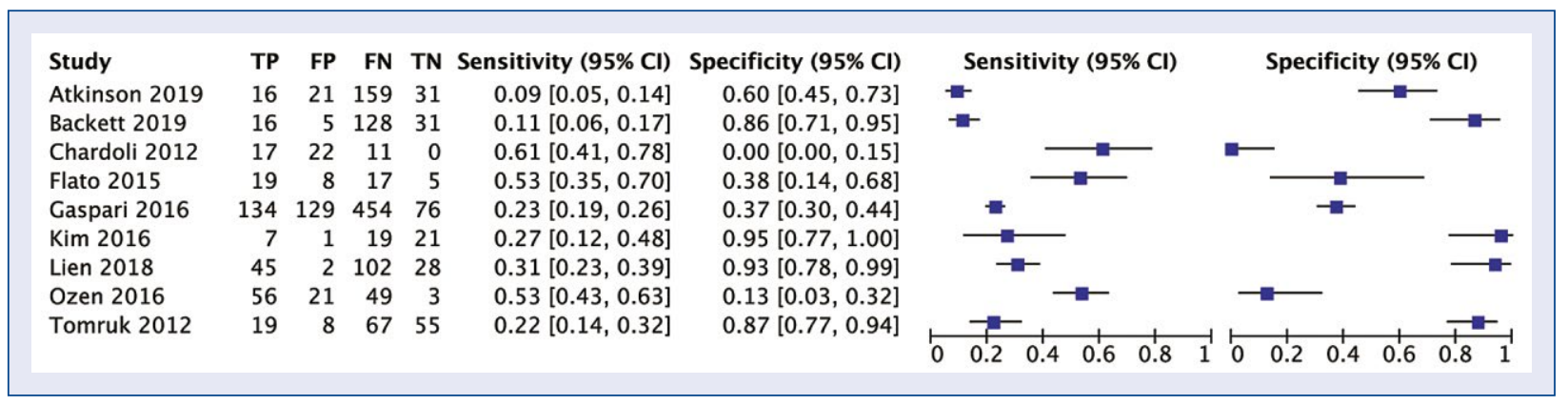

Figure 3. Forrest plot of the overall sensitivity and specificity of echocardiography for predicting the return of spontaneous circulation after cardiac arrest; $\mathrm{Cl}$ - confidence interval; TP — true positive; FP — false positive; FN — false negative; TN - true negative.

\begin{tabular}{|c|c|c|c|c|c|c|c|c|}
\hline Study & TP & FP & FN & TN & Sensitivity $(95 \% \mathrm{Cl})$ & Specificity (95\% CI) & Sensitivity $(95 \% \mathrm{CI})$ & Specificity $(95 \% \mathrm{Cl})$ \\
\hline Aichinger 2012 & 4 & 6 & 31 & 1 & $0.11[0.03,0.27]$ & $0.14[0.00,0.58]$ & & \\
\hline Atkinson 2019 & 7 & 21 & 159 & 11 & $0.04[0.02,0.08]$ & $0.34[0.19,0.53]$ & E & \\
\hline Backett 2019 & 7 & 14 & 148 & 11 & $0.05[0.02,0.09]$ & $0.44[0.24,0.65]$ & 툴 & \\
\hline Blaivas 2001 & 20 & 13 & 136 & 0 & $0.13[0.08,0.19]$ & $0.00[0.00,0.25]$ & 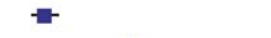 & \\
\hline Breitkreutz 2010 & 30 & 21 & 32 & 5 & $0.48[0.35,0.61]$ & $0.19[0.07,0.39]$ & & \\
\hline Cebicci 2014 & 74 & 7 & 324 & 5 & $0.19[0.15,0.23]$ & $0.42[0.15,0.72]$ & & \\
\hline Chua 2017 & 10 & 10 & 62 & 5 & $0.14[0.07,0.24]$ & $0.33[0.12,0.62]$ & - & \\
\hline Cureton 2012 & 6 & 14 & 141 & 1 & $0.04[0.02,0.09]$ & $0.07[0.00,0.32]$ & E & \\
\hline Gaspari 2016 & 76 & 187 & 492 & 38 & $0.13[0.11,0.16]$ & $0.17[0.12,0.22]$ & घ & \\
\hline Hayhurst 2011 & 11 & 9 & 29 & 1 & $0.28[0.15,0.44]$ & $0.10[0.00,0.45]$ & $\rightarrow-$ & \\
\hline Salen 2001 & 11 & 30 & 59 & 2 & $0.16[0.08,0.26]$ & $0.06[0.01,0.21]$ & $\rightarrow$ & $\rightarrow$ \\
\hline Salen 2005 & 8 & 3 & 59 & 0 & $0.12[0.05,0.22]$ & $0.00[0.00,0.71]$ & - & \\
\hline Schuster 2009 & 5 & 7 & 15 & 0 & $0.25[0.09,0.49]$ & $0.00[0.00,0.41]$ & & \\
\hline Tayal 2003 & 9 & 3 & 8 & 0 & $0.53[0.28,0.77]$ & $0.00[0.00,0.71]$ & $\frac{}{40.6} 0.81$ & 00 \\
\hline
\end{tabular}

Figure 4. Forrest plot of the overall sensitivity and specificity of echocardiography for survival to hospital admission after cardiac arrest; $\mathrm{Cl}$ - confidence interval; TP — true positive; FP — false positive; FN — false negative; TN true negative.

$0.139-0.260)$ and specificity 0.735 (95\% CI 0.589 $-0.851)$. Sensitivity and specificity of PoCUS in the case of IHCA was 0.166 (95\% CI $0.118-0.233)$ and -0.554 (95\% CI 0.425-0.677), respectively (Suppl. Table S3).
Fourteen studies reported survival to hospital admission [22-27, 29, 30, 32, 33, 37-40], in which sensitivity values ranged from 0.04 to 0.53 and specificity values that ranged from 0.00 to 0.44 (Fig. 4). The summary sensitivity and specificity 
values were 0.138 (95\% CI 0.122-0.155) and 0.201 (95\% CI $0.162-0.243$ ), respectively. The $\mathrm{Q}$ test revealed significant heterogeneity $(\mathrm{Q}=17.74$; $\mathrm{p}<0.001$ ), with substantial heterogeneity detected for sensitivity $\left(\mathrm{I}^{2}=91.2 \%\right.$; $\left.\mathrm{p}<0.001\right)$ and specificity $\left(\mathrm{I}^{2}=63.5 \% ; \mathrm{p}=0.003\right)$. The area under the ROC curve indicated low accuracy 0.1031 . PoCUS sensitivity and specificity for survival to admission after OHCA were 0.121 (95\% CI 0.093-0.154) and 0.261 (95\% CI 0.173-0.366), respectively.

\section{Discussion}

The present research analysis included studies that met the inclusion criteria. These studies included both OHCA and IHCA, and the patient population was varied. The current analysis included both retrospective studies, prospective clinical observational studies as well as observational and prospective series. Electrocardiographic findings in the analyzed cases were predominantly pulseless electrical activity and asystole.

Cardiac arrest, despite the development of therapeutic methods, is still a condition associated with a very high mortality rate [42-47]. One of the basic tasks during CPR is to shorten the resuscitation period and provide the fastest possible ROSC [48]. International guidelines recommend identifying and treating potentially reversible causes of SCA as soon as possible. With the lapse of time, the chances of victim survival decreases, whereas, with prolonged resuscitation, deterioration of a patient's neurological prognosis and fatigue of the medical personnel performing resuscitation, with possible deterioration in the quality of basic parameters related to chest compression are important factors $[49,50]$.

Ultrasonography has been used in intensive care for many years. Over the decades there has been an expansion in the use of ultrasound, the use of this method not only in diagnostic rooms but directly on the ward and even in the pre-hospital settings. Increasingly more physicians, as well as other medical staff, are trained and get experience in diagnosing life-threatening pathologies and the use of ultrasound equipment, which is widely available.

Ultrasound has many potential applications in CPR, ranging from the technical enhancement of resuscitation (correcting the correct position of the rescuer's hands) [51] to facilitating the identification of the correct cause of cardiac arrest [52], which is critical for further management. There are doubts about the prognostic potential of this technique for CPR; the results of studies in this aspect remain inconclusive. Ultrasonography can identify some potentially reversible causes of SCA, as well as assess myocardial contractility or the absence of any systolic activity of the heart. The complete absence of cardiac systolic activity is a poor prognostic factor for SCA.

The diagnostic aspect of ultrasonography in CPR is particularly relevant to selected special situations such as cardiac tamponade, pulmonary embolism, myocardial infarction, aortic dissection or rupture, hypovolemia, tension pneumothorax, papillary muscle rupture, it also enables the distinction of true asystole from ventricular fibrillation when there is doubt concerning the assessment of heart rhythm (e.g. artifacts, electrocardiogram muscle tremor) together with the assessment of myocardial contractile activity $[32,53]$. Studies on the use of ultrasonography indicate that $10-35 \%$ of patients with asystole demonstrate myocardial contractile activity.

Ultrasonography also allows the identification of arterial flow when there is doubt about the presence of a pulse on large arteries according to international guidelines. However, it is important to note that the presence of a pulse on large arteries is a prerequisite for the clinical exclusion of SCA - confirmation of the presence of a pulse providing minimal perfusion for survival with a good neurological prognosis [54].

A unique feature of this technique that is of great practical importance is the ability to recognize pseudo-pulseless electrical activity. PoCUS is of particular importance in cases of reversible causes of cardiac arrest; in such cases, it is also an ideal tool for performing emergency procedures under ultrasound guidance (e.g. cardiac tamponade decompression). Due to the advantages of the method, some authors consider the introduction of training also e.g. internal medicine physicians. An interesting development of the PoCUS technique is the use of both transthoracic (TTE) and transesophageal (TEE) echocardiograms. The advantage of this technique is that it can be used in real-time, continuously, and without interfering with ongoing resuscitation (TEE) or minimally interfering with ongoing CPR (TTE) [55].

A problem with the use of ultrasonography in SCA can be a lack of experience of the person performing the assessment and the interruption of resuscitation. With good training and coordination, this time can be reduced to a minimum [56]. Papers were published on the use of transesophageal ultrasonography, which allows continuous ultrasonographic assessment without the need for interruption of resuscitation efforts, although the 
accuracy of this assessment without interruptions in chest compressions has been questioned [57].

\section{Limitations of the study}

There are several limitations in this study. First, the total number of studies in our analyses was small; however, this may be offset by the moderate-to-large number of included patients $(\mathrm{n}=3265)$. Fifth, included studies did not assess all lung regions, as some patients were bedridden and posterior zones were difficult to be assessed. Moderate heterogeneity was found among the included studies, which was a result of differences in the study setting. Another limitation is the variety of staff preparation for ultrasound testing, as well as different medical staff performing PoCUS, starting with paramedics, through emergency physicians, emergency residents, and ending with the surgeons. Another limitation was the fact that not all studies reported onour primary outcome, which was survival to hospital discharge.

\section{Conclusions}

The results do not allow unambiguous recommendation of PoCUS as a predictor of resuscitation outcomes and further studies based on a large number of patients with full standardization of operators, their training and procedures performed are necessary.

\section{Acknowledgments}

The study was supported by the ERC Research Net and by the Polish Society of Disaster Medicine.

\section{Conflict of interest: None declared}

\section{References}

1. Price S, Uddin S, Quinn T. Echocardiography in cardiac arrest. Curr Opin Crit Care. 2010; 16(3): 211-215, doi: 10.1097/ MCC.0b013e3283399d4c, indexed in Pubmed: 20463464.

2. Kedan I, Ciozda W, Palatinus JA, et al. Prognostic value of pointof-care ultrasound during cardiac arrest: a systematic review. Cardiovasc Ultrasound. 2020; 18(1): 1, doi: 10.1186/s12947-0200185-8, indexed in Pubmed: 31931808.

3. Dudek M, Szarpak L, Ruetzler K. Application of interventional ultrasound in emergency medicine conditions. Disaster Emerg Med J. 2018; 3(4): 137-147, doi: 10.5603/demj.2018.0029.

4. Robak O, Dudek M, Ladny JR, et al. Cardiac tamponade as a cause of COVID-19. Cardiol J. 2020; 27(6): 900-901, doi: 10.5603/CJ.2020.0175, indexed in Pubmed: 33432570.

5. Liżewska-Springer A, Dąbrowska-Kugacka A, Lewicka E, et al. Echocardiographic predictors of atrial fibrillation recurrence after catheter ablation: A literature review. Cardiol J. 2020; 27(6): 848-856, doi: 10.5603/CJ.a2018.0067, indexed in Pubmed: 29924375.
6. Kurnicka K, Lichodziejewska B, Ciurzyński M, et al. Peak systolic velocity of tricuspid annulus is inferior to tricuspid annular plane systolic excursion for 30 days prediction of adverse outcome in acute pulmonary embolism. Cardiol J. 2020; 27(5): 558 -565, doi: 10.5603/CJ.a2018.0145, indexed in Pubmed: 30484266.

7. Querellou E, Leyral J, Brun C, et al. [In and out-of-hospital cardiac arrest and echography: a review]. Ann Fr Anesth Reanim. 2009; 28(9): 769-778, doi: 10.1016/j.annfar.2009.06.020, indexed in Pubmed: 19665861.

8. Blanco P, Volpicelli G. Common pitfalls in point-of-care ultrasound: a practical guide for emergency and critical care physicians. Crit Ultrasound J. 2016; 8(1): 15, doi: 10.1186/s13089-0160052-x, indexed in Pubmed: 27783380.

9. Aagaard R, Granfeldt A, Bøtker MT, et al. The right ventricle is dilated during resuscitation from cardiac arrest caused by hypovolemia: a porcine ultrasound study. Crit Care Med. 2017; 45(9): e963-e970, doi: 10.1097/CCM.0000000000002464, indexed in Pubmed: 28430698.

10. Konishi T, Funayama N, Yamamoto T, et al. Cerebral embolization from left atrial myxoma causing takotsubo cardiomyopathy complicated with congestive heart failure. Cardiol J. 2020; 27(4): 439-440, doi: 10.5603/CJ.2020.0118, indexed in Pubmed: 32929710.

11. Tsou PY, Kurbedin J, Chen YS, et al. Accuracy of point-of-care focused echocardiography in predicting outcome of resuscitation in cardiac arrest patients: A systematic review and meta-analysis. Resuscitation. 2017; 114: 92-99, doi: 10.1016/j.resuscitation.2017.02.021, indexed in Pubmed: 28263791.

12. Gulalp B, Evrin T, Akarca FK, et al. Point-of-Care emergency ultrasonography in non-traumatic cardiac arrest and near-arrest emergency patients; a pilot trial. Disaster Emerg Med J. 2020, doi: 10.5603/demj.a2020.0032.

13. Soar J, Berg K, Andersen L, et al. Adult Advanced Life Support: 2020 International Consensus on Cardiopulmonary Resuscitation and Emergency Cardiovascular Care Science with Treatment Recommendations. Resuscitation. 2020; 156: A80-A119, doi: 10.1016/j.resuscitation.2020.09.012.

14. Finn TE, Ward JL, Wu CTe, et al. COACHRED: A protocol for the safe and timely incorporation of focused echocardiography into the rhythm check during cardiopulmonary resuscitation. Emerg Med Australas. 2019; 31(6): 1115-1118, doi: 10.1111/17426723.13374, indexed in Pubmed: 31456338.

15. Moher D, Shamseer L, Clarke M, et al. PRISMA-P Group. Preferred reporting items for systematic review and meta-analysis protocols (PRISMA-P) 2015 statement. Syst Rev. 2015; 4: 1, doi: 10.1186/2046-4053-4-1, indexed in Pubmed: 25554246.

16. Sterne JAC, Hernán MA, Reeves BC, et al. ROBINS-I: a tool for assessing risk of bias in non-randomised studies of interventions. BMJ. 2016; 355: i4919, doi: 10.1136/bmj.i4919, indexed in Pubmed: 27733354.

17. Sterne JAC, Savović J, Page MJ, et al. RoB 2: a revised tool for assessing risk of bias in randomised trials. BMJ. 2019; 366: 14898, doi: 10.1136/bmj.14898, indexed in Pubmed: 31462531.

18. McGuinness LA, Higgins JPT. Risk-of-bias VISualization (robvis): An R package and Shiny web app for visualizing risk-of-bias assessments. Res Synth Methods. 2021; 12(1): 55-61, doi: 10.1002/jrsm.1411, indexed in Pubmed: 32336025.

19. Mantel N, Haenszel W. Statistical aspects of the analysis of data from retrospective studies of disease. J Natl Cancer Inst. 1959; 22(4): 719-748, indexed in Pubmed: 13655060.

20. DerSimonian R, Laird N, DerSimonian R, et al. Meta-analysis in clinical trials. Control Clin Trials. 1986; 7(3): 177-188, doi: 10.1016/0197-2456(86)90046-2, indexed in Pubmed: 3802833. 
21. Higgins JPT, Thompson SG, Deeks JJ, et al. Measuring inconsistency in meta-analyses. BMJ. 2003; 327(7414): 557-560, doi: 10.1136/bmj.327.7414.557, indexed in Pubmed: 12958120.

22. Atkinson PR, Beckett N, French J, et al. Does point-of-care ultrasound use impact resuscitation length, rates of intervention, and clinical outcomes during cardiac arrest? A study from the sonography in hypotension and cardiac arrest in the emergency department (shoc-ed) investigators. Cureus. 2019; 11(4): e4456, doi: 10.7759/cureus.4456, indexed in Pubmed: 31205842.

23. Aichinger G, Zechner PM, Prause G, et al. Cardiac movement identified on prehospital echocardiography predicts outcome in cardiac arrest patients. Prehosp Emerg Care. 2012; 16(2): 251-255, doi: 10.3109/10903127.2011.640414, indexed in Pubmed: 22235765 .

24. Beckett N, Atkinson P, Fraser J, et al. Do combined ultrasound and electrocardiogram-rhythm findings predict survival in emergency department cardiac arrest patients? The Second Sonography in Hypotension and Cardiac Arrest in the Emergency Department (SHoC-ED2) study. CJEM. 2019; 21(6): 739-743, doi: 10.1017/cem.2019.397, indexed in Pubmed: 31566175.

25. Blaivas M, Fox JC. Outcome in cardiac arrest patients found to have cardiac standstill on the bedside emergency department echocardiogram. Acad Emerg Med. 2001; 8(6): 616-621, doi: 10.1111/j.1553-2712.2001.tb00174.x, indexed in Pubmed: 11388936.

26. Breitkreutz R, Price S, Steiger HV, et al. Focused echocardiographic evaluation in life support and peri-resuscitation of emergency patients: a prospective trial. Resuscitation. 2010; 81(11): 1527-1533, doi: 10.1016/j.resuscitation.2010.07.013, indexed in Pubmed: 20801576.

27. Cebicci H, Salt O, Gurbuz S, et al. Benefit of cardiac sonography for estimating the early term survival of the cardiopulmonary arrest patients. Hippokratia. 2014; 18(2): 125-129, indexed in Pubmed: 25336874.

28. Chardoli M, Heidari F, Rabiee H, et al. Echocardiography integrated ACLS protocol versus conventional cardiopulmonary resuscitation in patients with pulseless electrical activity cardiac arrest. Chin J Traumatol. 2012; 15(5): 284-287, indexed in Pubmed: 23069099.

29. Chua MT, Chan GWh, Kuan WS. Reversible causes in cardiovascular collapse at the emergency department using ultrasonography (REVIVE-US). Ann Acad Med Singap. 2017; 46(8): 310-316, indexed in Pubmed: 28920131.

30. Cureton EL, Yeung LY, Kwan RO, et al. The heart of the matter: utility of ultrasound of cardiac activity during traumatic arrest. J Trauma Acute Care Surg. 2012; 73(1): 102-110, doi: 10.1097/ TA.0b013e3182569ebc, indexed in Pubmed: 22743379.

31. Flato UA, Paiva EF, Carballo MT, et al. Echocardiography for prognostication during the resuscitation of intensive care unit patients with non-shockable rhythm cardiac arrest. Resuscitation. 2015; 92: 1-6, doi: 10.1016/j.resuscitation.2015.03.024, indexed in Pubmed: 25891961.

32. Gaspari R, Weekes A, Adhikari S, et al. Emergency department point-of-care ultrasound in out-of-hospital and in-ED cardiac arrest. Resuscitation. 2016; 109: 33-39, doi: 10.1016/j.resuscitation.2016.09.018, indexed in Pubmed: 27693280.

33. Hayhurst C, Lebus C, Atkinson PR, et al. An evaluation of echo in life support (ELS): is it feasible? What does it add? Emerg Med J. 2011; 28(2): 119-121, doi: 10.1136/emj.2009.084202, indexed in Pubmed: 20921017.
34. Kim HB, Suh JY, Choi JH, et al. Can serial focussed echocardiographic evaluation in life support (FEEL) predict resuscitation outcome or termination of resuscitation (TOR)? A pilot study. Resuscitation. 2016; 101: 21-26, doi: 10.1016/j.resuscitation.2016.01.013, indexed in Pubmed: 26829701.

35. Lien WC, Hsu SH, Chong KM, et al. US-CAB protocol for ultrasonographic evaluation during cardiopulmonary resuscitation: Validation and potential impact. Resuscitation. 2018; 127: 125-131, doi: 10.1016/j.resuscitation.2018.01.051, indexed in Pubmed: 29410061.

36. Ozen C, Salcin E, Akoglu H, et al. Assessment of ventricular wall motion with focused echocardiography during cardiac arrest to predict survival. Turk J Emerg Med. 2016; 16(1): 12-16, doi: 10.1016/j.tjem.2015.08.001, indexed in Pubmed: 27239632.

37. Salen P, O'Connor R, Sierzenski P, et al. Can cardiac sonography and capnography be used independently and in combination to predict resuscitation outcomes? Acad Emerg Med. 2001; 8(6): 610-615, doi: 10.1111/j.1553-2712.2001.tb00172.x, indexed in Pubmed: 11388934.

38. Salen P, Melniker L, Chooljian C, et al. Does the presence or absence of sonographically identified cardiac activity predict resuscitation outcomes of cardiac arrest patients? Am J Emerg Med. 2005; 23(4): 459-462, doi: 10.1016/j.ajem.2004.11.007, indexed in Pubmed: 16032611.

39. Schuster KM, Lofthouse R, Moore C, et al. Pulseless electrical activity, focused abdominal sonography for trauma, and cardiac contractile activity as predictors of survival after trauma. J Trauma. 2009; 67(6): 1154-1157, doi: 10.1097/TA.0b013e3181c303e8, indexed in Pubmed: 20009660.

40. Tayal VS, Kline JA. Emergency echocardiography to detect pericardial effusion in patients in PEA and near-PEA states. Resuscitation. 2003; 59(3): 315-318, doi: 10.1016/s0300-9572(03)00245-4, indexed in Pubmed: 14659600.

41. Tomruk O, Erdur B, Cetin G, et al. Assessment of cardiac ultrasonography in predicting outcome in adult cardiac arrest. J Int Med Res. 2012; 40(2): 804-809, doi: 10.1177/147323001204000247, indexed in Pubmed: 22613446.

42. Umińska JM, Ratajczak J, Buszko K, et al. Impact of mild therapeutic hypothermia on bioavailability of ticagrelor in patients with acute myocardial infarction after out-of-hospital cardiac arrest. Cardiol J. 2020; 27(6): 780-788, doi: 10.5603/CJ.a2019.0024, indexed in Pubmed: 30799546.

43. Robak O, Pruc M, Malysz M, et al. Pre-filled syringes with adrenaline during cardiopulmonary resuscitation in nonshockable rhythms. Pilot randomised crossover simulation study. Disaster Emerg Med J. 2020; 5(2): 78-84, doi: 10.5603/demj.a2020.0025.

44. Szarpak L, Smereka J, Ruetzler K. Targeted temperature management: State of the Art. Disaster Emerg Med J. 2019; 4(2): 68-73, doi: 10.5603/demj.2019.0014.

45. Majer J, Jaguszewski MJ, Frass M, et al. Does the use of cardiopulmonary resuscitation feedback devices improve the quality of chest compressions performed by doctors? A prospective, randomized, cross-over simulation study. Cardiol J. 2019; 26(5): 529_ -535, doi: 10.5603/CJ.a2018.0091, indexed in Pubmed: 30155865.

46. Ludwin K, Smereka J, Jaguszewski M, et al. Place of magnesium sulfate in cardiopulmonary resuscitation. A systematic review and meta-analysis. Disaster Emerg Med J. 2020; 5(4): 182-189, doi: 10.5603/demj.a2020.0041.

47. Yilmaz E, Arsava EM, Topcuoglu MA. Resuscitation in COVID-19 patients: What do we know and what should we do? Car- 
diol J. 2020; 27(5): 656-657, doi: 10.5603/CJ.2020.0161, indexed in Pubmed: 33165906.

48. Nadolny K, Bujak K, Kucap M, et al. The silesian registry of out-of-hospital cardiac arrest: study design and results of a threemonth pilot study. Cardiol J. 2020; 27(5): 566-574, doi: 10.5603/ CJ.a2018.0140, indexed in Pubmed: 30444257.

49. Al-Jeabory M, Safiejko K, Bialka S, et al. Impact of COVID-19 on bystander cardiopulmonary resuscitation in out-of-hospital cardiac arrest: Is it as bad as we think? Cardiol J. 2020; 27(6): 884-885, doi: 10.5603/CJ.a2020.0179, indexed in Pubmed: 33346369.

50. Malysz M, Jaguszewski M, Szarpak L, et al. Comparison of different chest compression positions for use while wearing CBRN-PPE: a randomized crossover simulation trial. Disaster Emerg Med J. 2020, doi: 10.5603/demj.a2020.0034.

51. Zanatta M, Lorenzi C, Scorpiniti M, et al. Ultrasound-Guided chest compressions in out-of-hospital cardiac arrests. J Emerg Med. 2020; 59(6): e225-e233, doi: 10.1016/j.jemermed.2020.07.005, indexed in Pubmed: 32912645.

52. Devia Jaramillo G, Navarrete Aldana N, Rojas Ortiz Z. Rhythms and prognosis of patients with cardiac arrest, emphasis on pseudopulseless electrical activity: another reason to use ultrasound in emergency rooms in Colombia. Int J Emerg Med. 2020; 13(1): 62, doi: 10.1186/s12245-020-00319-4, indexed in Pubmed: 33276729.
53. Olszynski PA, Bryce R, Hussain Q, et al. Use of a simple ultrasound device to identify the optimal area of compression for out-of-hospital cardiac arrest. Cureus. 2021; 13(1): e12785, doi: 10.7759/cureus.12785, indexed in Pubmed: 33489641.

54. Sanchez S, Miller M, Asha S. Assessing the validity of two-dimensional carotid ultrasound to detect the presence and absence of a pulse. Resuscitation. 2020; 157: 67-73, doi: 10.1016/j.resuscitation.2020.10.002, indexed in Pubmed: 33058995.

55. Long B, Alerhand S, Maliel K, et al. Echocardiography in cardiac arrest: An emergency medicine review. Am J Emerg Med. 2018 36(3): 488-493, doi: 10.1016/j.ajem.2017.12.031, indexed in Pubmed: 29269162.

56. Chou EH, Wang CH, Monfort R, et al. Association of ultrasoundrelated interruption during cardiopulmonary resuscitation with adult cardiac arrest outcomes: A video-reviewed retrospective study. Resuscitation. 2020; 149: 74-80, doi: 10.1016/j.resuscitation.2020.02.004, indexed in Pubmed: 32068026.

57. Wang LM, Zhong Y, Ming-Hua Su, et al. Compression indexes measured by transthoracic echocardiographic might be not accurate without interrupting chest compressions. Crit Care. 2020; 24(1): 51, doi: 10.1186/s13054-020-2748-3, indexed in Pubmed: 32054501 . 